\title{
Oscillation of a Nonlinear Neutral Difference Equation with Positive and Negative Coefficients
}

\author{
Xilan Liu*, Dejie Deng and Huijuan Wang \\ Department of Mathematics and Statistics, Qinghai Nationalities University, Xining, China \\ Email: doclanliu@163.com
}

\begin{abstract}
This paper is concerned with the oscillatory behavior of a class of neutral nonlinear difference equation with positive and negative coefficients. The oscillation criteria are given, which extended the results of known ones.
\end{abstract}

Keywords: Neutral, nonlinear difference equation, positive and negative coefficient

\section{Introduction}

Oscillatory behavior is an important topic for difference equations, which has attracted many researchers for many years, see for example $[1,2,3,4,5,6]$ and references therein. In [5], the author discussed the oscillation property for a neutral linear difference equation with positive and negative coefficients

$$
\Delta[x(n)-r(n) x(n-\xi)]+p(n) x(n-\tau)-q(n) x(n-\sigma)=0, n=0,1,2, \ldots,
$$

where $\xi, \tau, \sigma$ are positive integers and that $\tau \geq \sigma, r(n)$ is a real sequence, $p(n)$ and $q(n)$ are nonnegative sequences. Note that (1) is linear. Motivated by [5], in this paper, we consider the oscillation of a class of nonlinear neutral difference equation with positive and negative coefficients

$$
\Delta[x(n)-R(n) x(n-r)]+P(n) f(x(n-k))-Q(n) f(x(n-l))=0, n \geqslant n_{0}
$$

where $R(n), P(n)$ and $Q(n)$ are nonnegative sequences, $r, k, l, n_{0}$ are positive integers with $k \geq l+1$, and $f \in C(R, R)$. To our knowledge, no any oscillation results for nonlinear equation (1) with positive and negative coefficients has been presented.

\section{Main Results}

We make the following assumptions:

(H1) $P(n), Q(n)$ and $R(n)$ are nonnegative sequences, $P(n)$ and $R(n)+Q(n)$ have positive subsequences;

(H2) $H(n):=P(n+k-l)-Q(n)$ is nonnegative and has a positive subsequence;

(H3) There exists a constant $A>0$ such that $|f(x)| \leq A|x|(x \in R), x f(x)>0$ for $x \neq 0, x \in \mathbf{R}$;

Let

$$
m_{0}:=\left\{\begin{array}{ll}
k, & R \equiv 0, \\
\max \{r, k\}, & \text { others; }
\end{array} \quad m_{1}:= \begin{cases}l, & R \equiv 0 \\
r, & Q \equiv 0, \\
\min \{r, l\}, & \text { others }\end{cases}\right.
$$

and

$$
z_{x}(n):=x(n)-R(n) x(n-r)-\sum_{i=n-k+l}^{n-1} P(i+k-l) f(x(i-l)), n \geqslant m_{0}+n_{0} .
$$

Consider the following inequality

$$
\Delta[x(n)-R(n) x(n-r)]+P(n) f(x(n-k))-Q(n) f(x(n-l)) \leqslant 0, n \geqslant n_{0} .
$$

We have 
Lemma 1 Assume that $\{x(n)\}$ is an eventual positive solution of (4). If

$$
R(n)+A \sum_{i=n-k+l}^{n-1} Q(i) \leq 1
$$

holds for $n$ large enough, then $\Delta z_{x}(n) \leq 0, z_{x}(n)>0$ for $n$ large enough.

Proof. Let $\{x(n)\}$ be a solution of the difference inequality (4). Then there exists $n_{1} \geq n_{0}$ such that $x\left(n-m_{0}\right)>0$ for $n \geq n_{1}$. $\mathrm{By}(4)$ and (3), we have

$$
\begin{aligned}
\Delta z_{x}(n) & =\Delta\left[x(n)-R(n) x(n-r)-\sum_{i=n-k+l}^{n-1} P(i+k-l) f(x(i-l))\right] \\
& =\Delta[x(n)-R(n) x(n-r)]-P(n+k-l) f(x(n-l))+P(n) f(x(n-k)) \\
& \leq-P(n+k-l) f(x(n-l))+Q(n) f(x(n-l)) \\
& \leq-H(n) f(x(n-l)) \leq 0 .
\end{aligned}
$$

So either $z_{x}(n)$ is eventually negative or $z_{x}(n)$ is eventually positive.

We first assume $z_{x}(n)$ is eventually negative. Then there exists $n_{2} \geq n_{1}, \alpha>0$ such that $z_{x}\left(n_{2}\right) \leq$ $-\alpha<0$ for $n \geq n_{2}$. Summing of two sides of (6) from $i=n_{2}$ to $n-1$ yields

$$
z_{x}(n) \leq z_{x}\left(n_{2}\right)-\sum_{i=n_{2}}^{n-1} H(i) f(x(i-l)) \leq-\alpha-\sum_{i=n_{2}}^{n-1} H(i) f(x(i-l)) .
$$

Thus for $n>n_{3} \geq n_{2}+m_{0}$, we have

$$
\begin{aligned}
x(n) & \leq-\alpha+R(n) x(n-r)+\sum_{i=n-k+l}^{n-1} P(i+k-l) f(x(i-l))-\sum_{i=n_{2}}^{n-1} H(i) f(x(i-l)) \\
& \leq-\alpha+R(n) x(n-r)+\sum_{i=n-k+l}^{n-1} Q(i) f(x(i-l)) .
\end{aligned}
$$

Accordingly, we consider the following two cases.

Case 1. $\{x(n)\}$ is unbounded. Then $\lim \sup x(n)=\infty$. Hence, there exists a subsequence $\left\{s_{j}\right\}_{j=1}^{\infty}$ such that $x\left(s_{j}\right) \rightarrow \infty$ as $s_{j} \rightarrow \infty$. Take $s_{j} \geq n_{3}+m_{0}$. Let $x\left(t_{j}\right)=\max \left\{x(n): n_{2} \leq n \leq s_{j}\right\}, j=1,2,3, \cdots$.

By (5) and (7), we have

$$
x\left(t_{j}\right) \leq-\alpha+R\left(s_{j}\right) x\left(t_{j}-r\right)+\sum_{i=t_{j}-k+l}^{t_{j}-1} Q(i) f(x(i-l)) \leq-\alpha+x\left(t_{j}\right),
$$

which is a contradiction.

Case 2. $\{x(n)\}$ is bounded. Then $\limsup _{n \rightarrow \infty} x(n)=a<\infty$, and there exists a subsequence $\left\{\bar{s}_{j}\right\}_{j=1}^{\infty}$ such that $\bar{s}_{j} \rightarrow \infty, x_{\bar{s}_{j}} \rightarrow a$ as $j \rightarrow \infty$. Let $\xi_{j}$ be the ones such that $x\left(\xi_{j}\right)=\max \left\{x_{s}: \bar{s}_{j}-m_{0} \leq s \leq \bar{s}_{j}-m_{1}\right\}$, $j=1,2,3, \cdots$. Thus $\xi_{j} \rightarrow \infty$ and $\limsup x\left(\xi_{j}\right) \leq a$ as $j \rightarrow \infty$.

By (5) and (7), we have

$$
x\left(\bar{s}_{j}\right) \leq-\alpha+R\left(\bar{s}_{j}\right) x\left(\bar{s}_{j}-r\right)+\sum_{i=\bar{s}_{j}-k+l}^{\bar{s}_{j}-1} Q(i) f(x(i-l)) \leq-\alpha+x\left(\xi_{j}\right),
$$

and further,

$$
a \leq-\alpha+\limsup _{j \rightarrow \infty} x\left(\xi_{j}\right) \leq-\alpha+a
$$

which is also a contradiction.

The above discussions finish the proof. 
Lemma 2. Assume that $\{x(n)\}$ is an eventually positive solution of the difference inequality (4). If

$$
R(n)+A \sum_{i=n-k+l}^{n-1} P(i+k-l) \geq 1
$$

holds for $n$ big enough, then either

$$
\liminf _{n \rightarrow \infty} x(n)>0
$$

or $z_{x}(n)<0$ holds for $n$ big enough.

Proof. Since $\{x(n)\}$ an eventually positive solution of the difference inequality (4), then there exists $n_{1} \geq n_{0}$ such that $x\left(n-m_{0}\right)>0$ for $n \geq n_{1}$. It is easy to know (6) holds. So there exits $n_{2} \geq n_{1}$ such that $z_{x}(n)<0$ or $z_{x}(n)>0$ is true for $n \geq n_{2}$.

Without loss the generality, we assume $z_{x}(n)>0$. Let $M:=\frac{1}{2} \min \left\{x(i): n_{2}-m_{0} \leq i \leq n_{2}\right\}$ Then $M \geq \frac{1}{2} z_{x}\left(n_{2}\right)>0$, and

$$
x(n)>\frac{1}{A} f(x(n))>M .
$$

for $n \geq n_{2}$. Indeed, if there exists $n_{3}>n_{2}$ such that $x(n)>\frac{1}{A} f(x(n))$ with $x(n) \leq M$ for $n \in\left[n_{2}, n_{3}\right)$. By (3) and (8), we have

$$
\begin{aligned}
M & \geq x\left(n_{3}\right)=z_{x}\left(n_{3}\right)+R\left(n_{3}\right) x\left(n_{3}-r\right)+\sum_{i=n_{3}-k+l}^{n_{3}-1} P(i+k-l) f(x(i-l)) \\
& >R\left(n_{3}\right) x\left(n_{3}-r\right)+\sum_{i=n_{3}-k+l}^{n_{3}-1} P(i+k-l) f(x(i-l)) \\
& >\left[R\left(n_{3}\right)+A \sum_{i=n_{3}-k+l}^{n_{3}-1} P(i+k-l)\right] M \geq M,
\end{aligned}
$$

which is a contradiction. So $\liminf _{n \rightarrow \infty} x(n)>0$.

By Lemma 1 and Lemma 2, we can obtain the following

Lemma 3. Assume that $\{x(n)\}$ is an eventually positive solution of the difference inequality (4). If (5) and (8) hold for $n$ big enough, then

$$
\liminf _{n \rightarrow \infty} x(n)>0 \quad \text { and } \quad \Delta z_{x}(n) \leq 0, \quad z_{x}(n)>0 .
$$

Similar to [5], we can easily obtain

Lemma 4. Assume conditions $H(1)-H(3)$ are satisfied for $n$ big enough, and (5) holds. Then any solution of (1) is oscillatory if and only if the difference inequity (4) has no eventually positive solution.

Theorem 1. Assume (5) and (8) hold eventually and

$$
\sum_{i}^{\infty} H(i)=\infty \text {. }
$$

Then all solutions of (1) are oscillatory.

Proof. We shall prove by the contradiction. Let $\{x(n)\}$ is an eventually positive solution of the difference inequality (4). Assume $x\left(n-m_{0}\right)>0$ for $n_{1} \geq n_{0}, n \geq n_{1}$. By (12), summing two sides of (6) from $i=n_{1}$ to $\infty$ leads to

$$
z_{x}\left(n_{1}\right) \geq \sum_{i=n_{1}}^{\infty} H(i) f(x(i-l))=\infty,
$$

which is contradictory to Lemma 3 . The proof is complete. 
Example 1. Consider the following difference equation

$$
\Delta[x(n)-r(n) x(n-r)]+p(n) \sin (x(n-k))-q(n) \sin (x(n-l))=0, n \geqslant n_{0},
$$

where $p(n)=2 n, q(n)=\frac{1}{n}, r(n)=\frac{(-1)^{n}+1}{n}$ and $H(n)=\frac{2(n+1)^{2}-3}{n}$. Taking $k-l=2$, then all conditions of Theorem 1 hold. So all solutions of (1) are oscillatory.

\section{Conclusion}

Based on the oscillatory property of the difference inequalities, this paper considered the oscillation behavior of a nonlinear neutral difference equation with positive and negative coefficients. Under some conditions, the difference equation discussed is oscillatory. Necessary oscillatory conditions for (1) will be considered later.

\section{Acknowledgement}

This is supported by the NNSF of China (11361047) and the NSF of Qinghai Province (2017-ZJ-908).

\section{References}

1. S. R. Grace and S. Dontha, "Oscillation of higher order neutral difference equations of mixed type," Dynam. Systems Appl., vol. 12, no. 3, pp. 521-532, 2002.

2. M. P. Chen and B. G. Zhang, "Oscillation and comparison theorems of difference equations with positive and negative coefficients." Bull.inst.math.acad.sinica, no. 4, pp. 295-306, 1994.

3. X. L. Liu, "Oscillatory behavior of third-order difference equations with asynchronous nonlinearities."

4. G. Ladas, "Oscillation of difference equations with positive and negative coefficients," Rocky Mountain J. Math., vol. 20, pp. 1051-1061, 1990.

5. S. C. W.T. Li, "On a neutral dfference equation with positive and negative coefficients," Southeast Asian Bull. Math., vol. 22, pp. 407-418, 1998.

6. X.L.Liu and Y.Y.Dong, "Oscillatory behavior of third-order difference equations with asynchronous nonlinearities," International J. Difference Eqs., vol. 9, pp. 223-231, 2014. 